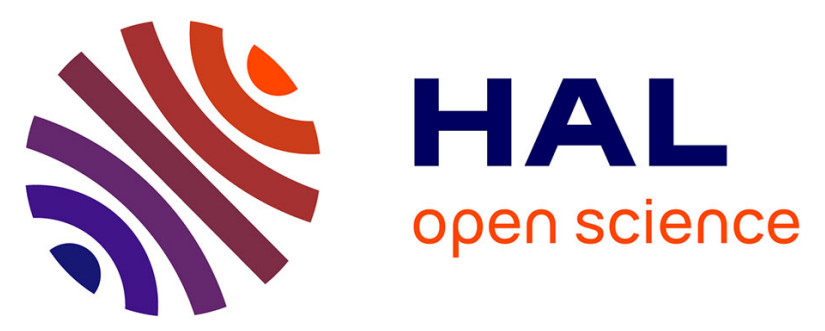

\title{
A Hybrid Adaptive Inverse for Uncertain SISO Linear Plants with Full Relative Degree*
}

Matteo Cocetti, Matteo Ragni, Sophie Tarbouriech, Luca Zaccarian

\section{To cite this version:}

Matteo Cocetti, Matteo Ragni, Sophie Tarbouriech, Luca Zaccarian. A Hybrid Adaptive Inverse for Uncertain SISO Linear Plants with Full Relative Degree *. 2019 American Control Conference (ACC), Jul 2019, Philadelphia, United States. pp.2315-2320, 10.23919/ACC.2019.8814785 • hal-03028110

\section{HAL Id: hal-03028110 https://hal.science/hal-03028110}

Submitted on 27 Nov 2020

HAL is a multi-disciplinary open access archive for the deposit and dissemination of scientific research documents, whether they are published or not. The documents may come from teaching and research institutions in France or abroad, or from public or private research centers.
L'archive ouverte pluridisciplinaire HAL, est destinée au dépôt et à la diffusion de documents scientifiques de niveau recherche, publiés ou non, émanant des établissements d'enseignement et de recherche français ou étrangers, des laboratoires publics ou privés. 


\title{
A Hybrid Adaptive Inverse for Uncertain SISO Linear Plants with Full Relative Degree*
}

\author{
Matteo Cocetti ${ }^{1}$, Matteo Ragni ${ }^{2}$, Sophie Tarbouriech ${ }^{1}$, Luca Zaccarian ${ }^{1}$
}

\begin{abstract}
We propose a hybrid adaptive feed-forward regulator for single-input single-output linear plants with full relative degree. The scheme includes an adaptive law that estimates the inverse of the plant and provides a feed-forward control calculated on the basis of the desired output and its derivatives. The adaptation is performed during discrete time events, called jumps, while the feed-forward action is continuous. This combination leads to a full hybrid system. The advantage of this framework is a conceptual separation between the adaptation dynamics, which is discrete, and the plant dynamics, which is continuous. Under an assumption of a persistence of excitation, we show through examples that the output asymptotically tracks the desired reference and that the estimate of the parameters of the inverse converges.
\end{abstract}

\section{INTRODUCTION}

From an abstract point of view, a dynamical system can be thought as an operator mapping an initial condition and an input function into an output. Usually, inputs and outputs are restricted to belong to some functional spaces a priori defined. Given the desired output (i.e. an element of the output space), the possibility of actually achieving it depends on the existence of its pre-image in the input space. Then, it appears quite natural to try to invert the operator and use the inverse to find the input that is mapped to the desired output.

This setting has been proposed for the first time by Roger Brockett in [1], where the concept of reproducibility has been introduced to denote the possibility for a dynamical system output to follow the desired trajectory.

A constructive solution for linear time invariant scalar systems has been developed by Brockett himself in [2], [3], employing the idea of inverse system. Intuitively the inverse of a dynamical system $\mathcal{H}$ is again a dynamical system $\mathcal{H}^{-1}$, but with swapped role of inputs and outputs. The inverse system $\mathcal{H}^{-1}$ uses the desired output for $\mathcal{H}$, and a certain number of derivatives, as inputs and produces as outputs the state and input trajectories for $\mathcal{H}$.

If the dynamical system $\mathcal{H}$ is known and admits a stable inverse, $\mathcal{H}^{-1}$ can be regarded as a generator of a feedforward control. This idea of inverse system and feedforward control has already been successfully applied in

\footnotetext{
*Work supported by grant PowerLyap funded by CaRiTRo and by Dana Rexroth Transmission Systems S.r.l.

${ }^{1}$ Matteo Cocetti, Sophie Tarbouriech, and Luca Zaccarian are with LAAS-CNRS, Université de Toulouse, CNRS, Toulouse, France \{matteo. cocetti, tarbour, zaccarian\}@laas.fr. Matteo Cocetti and Luca Zaccarian are also with Department of Industrial Engineering, University of Trento, Italy.

${ }^{2}$ Matteo Ragni is with Department of Industrial Engineering, University of Trento, via Sommarive, 9, 38123, Trento, Italy. matteo.ragni@unitn.it
}

various technological applications, such as nano-positioning control [4] and aerospace systems [5], [6], [7], [8]

From a theoretical perspective, inversion has then been further developed by Youla and Dorato [9], and by Silverman and Sain [10], [11], who provided a complete characterization of system invertibility in terms of controlled and conditioned invariant sub-spaces, and provided a constructive algorithm to compute an internally stable inverse.

The robustness aspect has been tackled by Wonham and Francis [12], [13], who realized that, restricting the class of possible reference signals for the plant, the problem of robust output regulation can be solved including a copy of the reference model in the loop, the so-called internal model. A decade later the internal model has been extended to the nonlinear setting by Isidori and Byrnes [14], who developed the so called Byrnes-Isidori equations, a set of algebraic partial differential equations that are necessary and sufficient for robust output regulation of nonlinear systems.

The concept of the inverse system gained some popularity also in the subsequent years, and many authors focused on obtaining internally stable inverses for non-minimum phase plants [15], [16], [17], [18], and for generic nonlinear systems [19], [20]. Also high-gain observers can be thought, up to a certain extent, as an approximate feedback linearization of the system [21], [22], and for this reason are usually combined with with state feedback control schemes to achieve output regulation.

More recently, the problem of obtaining stable inverse for uncertain systems, has been investigated using the notion of output to input stability (OIS), see [23]. This definition does not rely on any special normal form nor an explicit form of the zero dynamics, but captures the intuitive idea that for an invertible system, the internal state and the input are bounded by a suitable function of the output and its derivatives (modulo a decaying term depending on initial conditions). This notion is more suitable for robustness analysis, because does not involve changes of coordinates, and admits a Lyapunov characterization.

In this paper, we investigate the possibility to obtain an exact inverse model for a single-input single-output uncertain linear system. Uncertainties are compensated through an adaptive mechanism triggered at discrete-time instants called jumps. The continuous nature of the inverse model, and the discrete nature of the adaptation dynamics are combined using the hybrid system formalism. Solutions for more general classes of systems are available in the literature, but the novelty of this paper is in the hybrid adaptive law. This idea of using an inverse system to provide the correct feed- 
forward control and the possibility of discretely adapt the inverse to compensate for uncertainties has been inspired by [24], [25], where the authors use an adaptive feed-forward control in combination with a First Order Reset Element (FORE) [26] to control the output of a first order linear uncertain plant. Compared to [24], [25] in this paper we generalize the class of plants under consideration and we link this technique with inversion based control. Finally we propose a novel update law for the estimation of the inverse system.

Notation and preliminaries: We employ hybrid dynamical system of the form

$$
\mathcal{H}:=\left\{\begin{array}{l}
z \in C, \quad \dot{z} \in F(z) \\
z \in D, \quad z^{+} \in G(z),
\end{array}\right.
$$

where $z \in C \cup D$ is the state, $F, G: \mathbb{R}^{n} \rightrightarrows \mathbb{R}^{n}$ are outer semicontinuous set-valued maps, locally convex and bounded, (named respectively flow map and jump map) and $C, D$ are closed subsets of $\mathbb{R}^{n}$ (named flow set and jump set). In general $C \cap D \neq \emptyset$, allowing multiple solutions. Closeness of $C, D$ and outer-semicontinuity of $F, G$ ensure existence of solutions for [1], see [27, Assumption 6.5, Chap. 6]. Solutions are defined over a subset $E \subset \mathbb{R}_{\geq 0} \times \mathbb{N}$ called hybrid time domain. A hybrid time domain is the union of finitely or infinitely many intervals $\left[t_{j}, t_{j+1}\right] \times\{j\}$, where $0=t_{0} \leq t_{1} \leq t_{2} \leq \ldots$, with the last interval possibly of the form $\left[t_{j}, t_{j+1}\right)$ or $\left[t_{j}, \infty\right)$. The instants $t_{j}$ are called jump times. A hybrid arc is a function $\phi: \operatorname{dom} \phi \rightarrow \mathbb{R}^{n}$, where the domain $\operatorname{dom} \phi$ of $\phi$ is a hybrid time domain and, if $I_{j}(\phi):=\{t:(t, j) \in \operatorname{dom} \phi\}$ has nonempty interior, then $t \mapsto \phi(t, j)$ is locally absolutely continuous on $I_{j}$. A hybrid $\operatorname{arc} \phi$ is a solution to (1) if $\phi(0,0) \in C \cup D$, and, if $I_{j}(\phi)$ has nonempty interior $\operatorname{int} I_{j}(\phi)$, then $\phi(t, j) \in C$ for all $t \in \operatorname{int} I_{j}(\phi)$ and $\frac{\mathrm{d}}{\mathrm{d} t} \phi(t, j) \in F(\phi(t, j))$ for almost all $t \in I_{j}(\phi)$. If $(t, j) \in \operatorname{dom} \phi$ and $(t, j+1) \in \operatorname{dom} \phi$ then $\phi(t, j) \in D$ and $\phi(t, j+1) \in G(\phi(t, j))$. We say that a solution $\phi: \operatorname{dom} \phi \rightarrow \mathbb{R}^{n}$ is maximal if it cannot be extended, and complete if $\operatorname{dom} \phi$ is unbounded. Let $\mathcal{A} \subset \mathbb{R}^{n}$ be closed. The set $\mathcal{A}$ is Uniformly Globally Stable (UGS) if there exists a class $\mathcal{K}_{\infty}$ function $\alpha$ such that any solution $\phi$ to $\mathcal{H}$ satisfies $|\phi(t, j)|_{\mathcal{A}} \leq \alpha\left(|\phi(0,0)|_{\mathcal{A}}\right)$ for all $(t, j) \in \operatorname{dom} \phi$; where $|\phi|_{\mathcal{A}}:=\inf _{a \in \mathcal{A}}(|\phi-a|)$. Moreover, the set $\mathcal{A}$ is Uniformly Globally Asymptotically Stable (UGAS), if there exists a class $\mathcal{K} \mathcal{L}$ function $\beta$ such that any solution $\phi$ to $\mathcal{H}$ satisfies $|\phi(t, j)|_{\mathcal{A}} \leq \beta\left(|\phi(0,0)|_{\mathcal{A}}, t\right)$ for all $(t, j) \in \operatorname{dom} \phi$. The set $\mathcal{A}$ is Globally Exponentially Stable (GES) for $\mathcal{H}$, if it is UGAS and $\beta$ is of the form $\beta(x, t)=k \exp (-\lambda t) x$ for some positive constants $k, \lambda>0$.

\section{Construction of the inverse}

In this section, we recall a few results to invert a singleinput single-output linear system. We start by considering a plant described by a set of differential equations of the form

$$
\left\{\begin{array}{l}
\dot{x}=A x+B u \\
y=C x
\end{array}\right.
$$

where $x \in \mathbb{R}^{n}$ is the state, $u \in \mathbb{R}$ is the input and $y \in \mathbb{R}$ is the output. We stress that the matrices $A, B, C$ are not known, but we assume that they satisfy the following assumption.

Assumption 1: The triple $(C, A, B)$ is minimal, internally stable and with full relative degree.

The full relative degree ensures that the inverse system of (2) has no zero dynamics (and is minimum phase). Moreover the desired feed-forward input can be obtained by taking linear combinations of the desired output and its derivatives. The full relative degree assumption implies the following:

$$
\begin{array}{r}
C B=C A B=\cdots=C A^{n-2} B=0, \\
C A^{n-1} B \neq 0 .
\end{array}
$$

To derive the inverse system, let us consider a copy of 22 of the following form:

$$
\left\{\begin{array}{l}
\dot{x}_{\mathrm{d}}=A x_{\mathrm{d}}+B u_{\mathrm{d}} \\
y_{\mathrm{d}}=C x_{\mathrm{d}} .
\end{array}\right.
$$

Here $x_{\mathrm{d}} \in \mathbb{R}^{n}$ is the desired state trajectory, while $u_{\mathrm{d}} \in \mathbb{R}$ and $y_{\mathrm{d}} \in \mathbb{R}$ are the corresponding input and output functions. The goal is to isolate $x_{\mathrm{d}}$ and $u_{\mathrm{d}}$ as functions of the output $y_{\mathrm{d}}$ and its derivatives. Differentiating the output of (4) until the input $u_{\mathrm{d}}$ appears explicitly, we obtain the following chain of equalities

$$
\left\{\begin{aligned}
y_{\mathrm{d}}^{(0)} & =C x_{\mathrm{d}} \\
& \vdots \\
y_{\mathrm{d}}^{(n)} & =C A^{n} x_{\mathrm{d}}+C A^{n-1} B u_{\mathrm{d}}
\end{aligned}\right.
$$

where we used 3$\}$ and we defined $y_{\mathrm{d}}^{(i)}:=\frac{\mathrm{d}^{i}}{\mathrm{~d} t^{i}} y_{\mathrm{d}}$.

Remark 1: According to Assumption 1 we need to differentiate $n$ times before the input $u_{\mathrm{d}}$ appears explicitly.

From the last equality in (5) we can easily obtain $u_{\mathrm{d}}$ as a function of $x_{\mathrm{d}}$ and $y_{\mathrm{d}}$, that results

$$
u_{\mathrm{d}}=\frac{y_{\mathrm{d}}^{(n)}}{C A^{n-1} B}-\frac{C A^{n} x_{\mathrm{d}}}{C A^{n-1} B} .
$$

Plugging (6) into (4) and collecting a few terms we obtain the so-called inverse system of (4),

$$
\left\{\begin{array}{l}
\dot{x}_{\mathrm{d}}=\left(A-\frac{B C A^{n}}{C A^{n-1} B}\right) x_{\mathrm{d}}+\frac{B}{C A^{n-1} B} y_{\mathrm{d}}^{(n)} \\
u_{\mathrm{d}}=-\frac{C A^{n}}{C A^{n-1} B} x_{\mathrm{d}}+\frac{1}{C A^{n-1} B} y_{\mathrm{d}}^{(n)} .
\end{array}\right.
$$

The inverse system (7) receives as input the $n$-th derivative of the desired output $y_{\mathrm{d}}$ and provides the corresponding feedforward control action $u_{\mathrm{d}}$ to achieve it. Because the matrices $A, B, C$ are not known, also the inverse system (7) is in general not known. Moreover, the representation (7) is not convenient because the unknown matrices appear everywhere in the dynamics. A more convenient parametrization for (7) can be obtained considering the change of coordinates defined by the observability matrix $O:=\left(C^{\top}, \ldots,\left(A^{n-1}\right)^{\top} C^{\top}\right)^{\top} \in$ $\mathbb{R}^{n \times n}$. The change of coordinates is well defined because by Assumption 1 the plant is minimal and square. Using the matrix $O$ we realize that the desired state $x_{\mathrm{d}}$ has a one to 
one correspondence with $y_{\mathrm{d}}$ and its derivatives up to order $n-1$ as follows,

$$
\left(y_{\mathrm{d}}^{(0)}, \ldots, y_{\mathrm{d}}^{(n-1)}\right)=O x_{\mathrm{d}}
$$

Using (8) and plugging it into (6), we obtain the desired feed-forward input $u_{\mathrm{d}}$ as a linear combination of $y_{\mathrm{d}}$ and its derivatives,

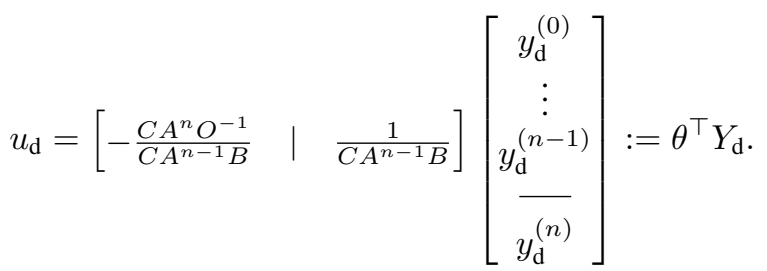

It is worth to notice that now all the uncertainties are concentrated in the parameters vector $\theta \in \mathbb{R}^{n+1}$. For convenience of notation we defined the vector $Y_{\mathrm{d}} \in \mathbb{R}^{n+1}$ collecting $y_{\mathrm{d}}$ and its derivatives up to order $n$.

Assumption 2: The function $Y_{\mathrm{d}}: \mathbb{R} \rightarrow \mathbb{R}^{n+1}$ is available, globally bounded by a constant $M>0$, and smooth.

If $\theta$ was available, the feed-forward input 9 would force $\lim _{t \rightarrow \infty} y_{\mathrm{d}}(t)-y(t)=0$, achieving asymptotic tracking. Because the vector $\theta$ is not available, we propose an adaptive feed-forward of the following form

$$
u=\hat{\theta}^{\top} Y_{\mathrm{d}},
$$

where $\hat{\theta} \in \mathbb{R}^{n+1}$ is an estimate of $\theta$. In the next section we propose a discrete mechanism that estimates $\hat{\theta}$. This algorithm, together with $(10)$ and the plant (2), results into a Uniformly Globally Stable (UGS) origin in proper error coordinates. Moreover, in case of sufficiently rich references, we believe that UGS can be strengthened to Uniform Global Asymptotic Stability (UGAS), which implies asymptotic output tracking.

\section{AdAPTATION: A SIMPLIFIED SETUP}

We start by considering a simplified setup where the output of the plant $y$ and all its derivative are available for feedback. Let us consider the following system

$$
\left\{\begin{array}{l}
\dot{x}=A x+B u \\
Y=C_{Y} x+D_{Y} u
\end{array}\right.
$$

where $Y:=\left(y^{(0)}, \ldots, y^{(n)}\right) \in \mathbb{R}^{n+1}$ and the matrices $C_{Y} \in$ $\mathbb{R}^{(n+1) \times n}, D_{Y} \in \mathbb{R}^{(n+1) \times 1}$ are defined below

$$
\left[C_{Y} \mid D_{Y}\right]=\left[\begin{array}{c|c}
C & 0 \\
C A & 0 \\
\vdots & \vdots \\
C A^{n-1} & 0 \\
C A^{n} & C A^{n-1} B
\end{array}\right]
$$

For (11) we choose a feed-forward input of the form (10), where $\theta \in \mathbb{R}^{n+1}$ is the vector containing the $n+1$ parameter defining the inverse of 11 . For $\hat{\theta}$ we propose a discrete update law of the following form

$$
\hat{\theta}^{+}=\hat{\theta}-\gamma \frac{Y\left(Y-Y_{\mathrm{d}}\right)^{\top}}{\max \left\{Y^{\top} Y, \epsilon^{2}\right\}} \hat{\theta},
$$

where $\gamma \in(0,2)$ is a positive scalar gain, tuning the adaptation speed, and $\epsilon \in \mathbb{R}$ a small quantity preventing the denominator from approaching zero. Continuous and discrete-time parameters estimation is a well-studied subject in the literature and many solutions are available, see for example [28] for an overview. However, the setup that we consider in this paper is different, because it combines a continuous-time plant and feed-forward control with a discrete-time estimator, leading to a full hybrid system. This setup can be better linked with the recent works of output regulation with external models and hybrid identifiers, see for example [29], [30] and [31].

Remark 2: The max term in (12) avoids divisions by zero when $Y^{\top} Y=0$. Moreover, it has the beneficial effect of slowing down the adaptation when $Y^{\top} Y$ is not sufficiently large.

The update law 12 is triggered by a timer $\tau \in \mathbb{R}_{\geq 0}$, which enforces a direct and a reverse dwell-time condition. Combining (11), (10), (12) and the timer $\tau$ we obtain a hybrid system reported below

$$
\begin{aligned}
& {\left[\begin{array}{l}
x \\
\hat{\theta} \\
\tau
\end{array}\right] \in\left[\begin{array}{c}
\mathbb{R}^{n} \\
\mathbb{R}^{n+1} \\
{\left[0, \tau_{\text {max }}\right]}
\end{array}\right], \quad\left\{\begin{array}{l}
\dot{x}=A x+B \hat{\theta}^{\top} Y_{\mathrm{d}} \\
\dot{\hat{\theta}}=0 \\
\dot{\tau}=1
\end{array}\right.} \\
& {\left[\begin{array}{l}
x \\
\hat{\theta} \\
\tau
\end{array}\right] \in\left[\begin{array}{c}
\mathbb{R}^{n} \\
\mathbb{R}^{n+1} \\
{\left[\tau_{\min }, \tau_{\max }\right]}
\end{array}\right], \quad\left\{\begin{array}{l}
x^{+}=x \\
\hat{\theta}^{+}=\hat{\theta}-\gamma \frac{Y\left(Y-Y_{\mathrm{d}}\right)^{\top}}{\max \left\{Y^{\top} Y, \epsilon^{2}\right\}} \hat{\theta} \\
\tau^{+}=0
\end{array}\right.}
\end{aligned}
$$

together with the output equation $Y=C_{Y} x+D_{Y} \hat{\theta}^{\top} Y_{\mathrm{d}}$.

The timer $\tau$ is designed as in [32], and works as a trigger for the jumps, in such a way that between two consecutive jumps there is always a minimum flow of ordinary time $\tau_{\text {min }} \in \mathbb{R}_{>0}$ and a maximum flow of $\tau_{\max } \in \mathbb{R}_{>0}$.

Formally, denoting by $t_{j}$ the jump instants $\left(j \in \mathbb{N}_{\geq 0}\right)$, the following holds $\tau_{\text {min }} \leq\left|t_{j+1}-t_{j}\right| \leq \tau_{\max }, \forall j \in \mathbb{N}_{\geq 0}$. Sequences of jumps that satisfy the above condition are rather general, and comprises virtually any possible sequence that is relevant from a practical viewpoint. For the stability analysis, let us consider the following change of coordinates, which highlights the error dynamics

$$
\left\{\begin{array}{l}
\tilde{x}=x-x_{\mathrm{d}} \\
\tilde{\theta}=\hat{\theta}-\theta .
\end{array}\right.
$$

Plugging 14 into 13 we obtain the following expression for the error

$$
\left\{\begin{array} { l } 
{ \dot { \tilde { x } } \in F ( \tilde { x } , \tilde { \theta } ) } \\
{ \dot { \tilde { \theta } } = 0 } \\
{ \dot { \tau } = 1 }
\end{array} \quad \left\{\begin{array}{l}
\tilde{x}^{+}=\tilde{x} \\
\tilde{\theta}^{+} \in G(\tilde{x}, \tilde{\theta}) \\
\tau^{+}=0
\end{array}\right.\right.
$$

together with the output equation $Y=Y_{\mathrm{d}}+C_{Y} \tilde{x}+D_{Y} \tilde{\theta}^{\top} Y_{\mathrm{d}}$. For compactness of notation, we omitted the flow and the jump sets that are anyway unaffected by (14). The multivalued maps $F: \mathbb{R}^{2 n+1} \rightrightarrows \mathbb{R}^{n}$ and $G: \mathbb{R}^{2 n+1} \rightrightarrows \mathbb{R}^{n+1}$ 
are defined as follows

$$
\begin{aligned}
& F(\tilde{x}, \tilde{\theta}):=\bigcup_{\left|Y_{\mathrm{d}}\right| \leq M} A \tilde{x}+B \tilde{\theta}^{\top} Y_{\mathrm{d}}, \\
& G(\tilde{x}, \tilde{\theta}):=\bigcup_{\left|Y_{\mathrm{d}}\right| \leq M}\left(I-\gamma \frac{Y Y^{\top}}{\max \left\{Y^{\top} Y, \epsilon^{2}\right\}}\right) \tilde{\theta},
\end{aligned}
$$

in this way 15 is autonomous, and Lyapunov theory applies.

Remark 3: The error system (15) posses a remarkable structure; during flow $\tilde{\theta}$ remains constant and $\tilde{x}$ changes, while during jumps $\tilde{x}$ remains constant and $\tilde{\theta}$ is updated. From the expression of the update law (12) we recognize that, if $Y$ is sufficiently rich (this idea will be formalized better in Definition 1, then $Y Y^{\top}$ spans all directions of $\mathbb{R}^{n+1}$, and $|\tilde{\theta}|$ converges to zero.

However, even when $Y Y^{\top}$ has no special properties, we can still prove that the set $\mathcal{A}:=\{0\} \times\{0\} \times\left[0, \tau_{\text {max }}\right]$ is uniformly globally stable (UGS) for 15 .

Proposition 1: Under Assumptions 1, 2, and for any $\gamma \in$ $(0,2)$ the attractor $\mathcal{A}$ is UGS for 15$)$.

Proof: Let us start by focusing on the $\tilde{\theta}$ dynamics. Consider the Lyapunov function $V_{\theta}(\tilde{\theta}):=\gamma^{-1} \tilde{\theta}^{\top} \tilde{\theta}$. The variation along the flow results $\left\langle\nabla V_{\theta}, \tilde{\theta}\right\rangle=0$, while during jumps yields

$$
V_{\theta}\left(\tilde{\theta}^{+}\right)-V_{\theta}(\tilde{\theta}) \leq-(2-\gamma) \tilde{\theta}^{\top} \frac{Y Y^{\top}}{\max \left\{Y^{\top} Y, \epsilon^{2}\right\}} \tilde{\theta} \leq 0 .
$$

We conclude that for any $\gamma \in(0,2)$ the Lyapunov function $V_{\theta}$ does not increase and the origin for the $\tilde{\theta}$ dynamics is UGS, moreover it satisfies $|\tilde{\theta}(t, j)| \leq|\tilde{\theta}(0,0)|$ for all $(t, j)$. Because $Y_{\mathrm{d}}$ and $\tilde{\theta}$ are globally bounded and the matrix $A$ is Hurwitz by Assumption 1, there are positive constants $c_{1}, c_{2}>0$, such that the following holds $|\tilde{x}(t, j)| \leq$ $c_{1} \mathrm{e}^{-\lambda_{1} t}|\tilde{x}(0,0)|+c_{2}\left|Y_{\mathrm{d}}\right||\tilde{\theta}(0,0)|$, for all $(t, j)$. We are finally in the position of showing that $\mathcal{A}$ is uniformly globally stable for (15). Applying the definition of UGS we derive the following $\mathcal{K}_{\infty}$ bound

$$
\begin{aligned}
\left|\left[\begin{array}{c}
\tilde{x}(t, j) \\
\tilde{\theta}(t, j) \\
\tau(t, j)
\end{array}\right]\right|_{\mathcal{A}}^{2} & =\left|\left[\begin{array}{c}
\tilde{x}(t, j) \\
\tilde{\theta}(t, j)
\end{array}\right]\right|^{2} \leq\left(c_{1} \mathrm{e}^{-\lambda_{1} t}|\tilde{x}(0,0)|+\right. \\
& \left.c_{2}\left|Y_{\mathrm{d}}\right||\tilde{\theta}(0,0)|\right)^{2}+|\tilde{\theta}(0,0)|^{2} \\
& \leq 2 c_{1}^{2}|\tilde{x}(0,0)|^{2}+\left(2 c_{2}^{2}\left|Y_{\mathrm{d}}\right|^{2}+1\right)|\tilde{\theta}(0,0)|^{2} \\
& \leq \max \left\{2 c_{1}^{2}, 2 c_{2}^{2} M^{2}+1\right\}\left|\left[\begin{array}{c}
\tilde{x}(0,0) \\
\tilde{\theta}(0,0) \\
\tau(0,0)
\end{array}\right]\right|_{\mathcal{A}}^{2},
\end{aligned}
$$

where we used the fact that $\left|Y_{\mathrm{d}}\right| \leq M$, see Assumption 2 .

Remark 4: Uniform global stability of $\mathcal{A}$ is not sufficient to conclude robustness. To enforce the missing uniform attractivity property we need additional properties for the signal $Y$.

Definition $1(P E)$ : A hybrid signal $Y$ is said to be persistently exciting at jumps $t_{j}$ if for the sequence $\left\{Y\left(t_{j}, j\right)\right\}_{j=0}^{\infty}$ there exist positive constants $\alpha_{1}, \alpha_{2}>0$ and $N \in \mathbb{N}_{\geq 1}$ such that

$$
0<\alpha_{1} I \leq \sum_{i=j}^{j+N} \frac{Y(t, i) Y(t, i)^{\top}}{Y(t, i)^{\top} Y(t, i)} \leq \alpha_{2} I, \quad \forall j \in \mathbb{N}_{\geq 0} .
$$

Persistence of excitation might be the missing ingredient to prove Uniform Global Asymptotic Stability (UGAS). This aspect is not discussed here, and will be explored in greater depth in future works. We proceed instead by presenting a more realistic scenario, where only the plant output $y$ is measured and used for control/adaptation.

\section{ADAPTATION: FULL SETUP}

In this section, we consider a more realistic scenario where the only measured quantity is the plant output $y$. As in classical adaptive control, we use a filtered version of the input-output behavior of the plant to identify its inverse. For this purpose, we consider two identical filters of order $n+1$, defined by the pair $(\Lambda, \Phi)$, that for simplicity we assume satisfies the following:

Assumption 3: The pair $(\Lambda, \Phi)$ is internally stable and in controllable canonical form.

For the filters we introduce two additional states $\sigma \in \mathbb{R}^{n+1}$ and $\eta \in \mathbb{R}^{n+1}$, representing respectively a filtered version of the plant output $y$ and plant input $u$. The two filters obey the following continuous/discrete dynamics

$$
\left\{\begin{array} { l } 
{ \dot { \sigma } = \Lambda \sigma + \Phi C x } \\
{ \dot { \eta } = \Lambda \eta + \Phi \hat { \theta } ^ { \top } Y _ { \mathrm { d } } }
\end{array} \quad \left\{\begin{array}{l}
\sigma^{+}=\sigma \\
\eta^{+}=\eta
\end{array}\right.\right.
$$

and the adaptive law $[12$ is modified accordingly as follows,

$$
\hat{\theta}^{+}=\hat{\theta}-\gamma \frac{\sigma}{\max \left\{\sigma^{\top} \sigma, \epsilon^{2}\right\}}\left(\sigma^{\top} \hat{\theta}-e_{1}^{\top} \eta\right),
$$

where $e_{1}^{\top}=(1,0, \ldots, 0) \in \mathbb{R}^{n+1}$ is the first element of the natural basis, and $\gamma \in(0,2)$. The modified law 19 does not require anymore the knowledge of the output $y$ and its derivatives, but a filtered version of the input output behavior is sufficient. Combining the update law (19) together with the filters $(18)$, the plant (2) and the feed-forward control (10) we obtain a hybrid system similar to (13). Then combining the previous change of coordinates $(14)$ and the one reported below for the filters $\tilde{\sigma}(t, j)=\sigma(t, j)-\int_{0}^{t} \mathrm{e}^{\Lambda(t-\xi)} \Phi C x(\xi, j) \mathrm{d} \xi$, and $\tilde{\eta}(t, j)=\eta(t, j)-\int_{0}^{t} \mathrm{e}^{\Lambda(t-\xi)} \Phi \hat{\theta}^{\top}(\xi, j) Y_{\mathrm{d}}(\xi, j) \mathrm{d} \xi$, we obtain the following representation for the closed-loop system in the error coordinates

$$
\left\{\begin{array} { l } 
{ \dot { \tilde { x } } \in F ( \tilde { x } , \tilde { \theta } ) } \\
{ \dot { \tilde { \sigma } } = \Lambda \tilde { \sigma } } \\
{ \dot { \tilde { \eta } } = \Lambda \tilde { \eta } } \\
{ \dot { \tilde { \theta } } = 0 } \\
{ \dot { \tau } = 1 }
\end{array} \quad \left\{\begin{array}{l}
\tilde{x}^{+}=\tilde{x} \\
\tilde{\sigma}^{+}=\tilde{\sigma} \\
\tilde{\eta}^{+}=\tilde{\eta} \\
\tilde{\theta}^{+}=\left(I-\gamma \frac{\sigma \sigma^{\top}}{\max \left\{\sigma^{\top} \sigma, \epsilon^{2}\right\}}\right) \tilde{\theta} \\
\quad-\gamma \frac{\sigma\left(\tilde{\sigma}^{\top} \theta-e_{1}^{\top} \tilde{\eta}\right)}{\max \left\{\sigma^{\top} \sigma, \epsilon^{2}\right\}} \\
\tau^{+}=0
\end{array}\right.\right.
$$

Remark 5: In 20) to keep the notation more compact, we did not fully substitute $\sigma$ with $\tilde{\sigma}$ and $\theta$ with $\tilde{\theta}$. 
Remark 6: The structure of (20) is similar to (15), except for the presence of two stable autonomous dynamics representing the contribution due to non-zero initial conditions of the filters (18). As we can see from (20) the $\tilde{\sigma}, \tilde{\eta}$ dynamics provide an exponentially decaying perturbation on the $\tilde{\theta}$ dynamics, but despite this, the main idea of the scheme is preserved, and a result similar to Proposition 1 holds.

Proposition 2: Under Assumptions 1, 2, 3 and for any $\gamma \in(0,2)$ the attractor $\mathcal{A}:=\{0\} \times\{0\} \times\{0\} \times\{0\} \times\left[0, \tau_{\max }\right]$ is UGS for 20.

Proof: We follow the same line of the proof of Proposition 1 . The difference with respect to the aforementioned proof is the presence of exponentially decaying perturbations on the $\tilde{\theta}$ dynamics. For this reason we start by showing that $|\tilde{\theta}(t, j)|$ is globally bounded by a $\mathcal{K}_{\infty}$ function depending on $|\tilde{\theta}(0,0)|,|\tilde{\sigma}(0,0)|$, and $|\tilde{\eta}(0,0)|$ for all $(t, j) \in \operatorname{dom} \tilde{\theta}$. According to Assumption 3 , the following exponential bound holds

$$
\left|\left[\begin{array}{c}
\tilde{\sigma}(t, j) \\
\tilde{\eta}(t, j)
\end{array}\right]\right| \leq c_{3} \mathrm{e}^{-\lambda_{2} t},\left|\left[\begin{array}{c}
\tilde{\sigma}(0,0) \\
\tilde{\eta}(0,0)
\end{array}\right]\right|,
$$

where $c_{3}$ and $\lambda_{2}$ are positive constants. Evaluating (21) at $\left(t_{j}, j\right)$ and $\left(t_{j-1}, j-1\right)$, and recalling that $t_{j}-t_{j-1} \geq \tau_{\min }$ (the dwell time condition imposed by the timer $\tau$ ), we derive the following recursion

$$
\left|\left[\begin{array}{l}
\tilde{\sigma}\left(t_{j}, j\right) \\
\tilde{\eta}\left(t_{j}, j\right)
\end{array}\right]\right| \leq \mathrm{e}^{-\lambda_{2} \tau_{\min }}\left|\left[\begin{array}{l}
\tilde{\sigma}\left(t_{j-1}, j-1\right) \\
\tilde{\eta}\left(t_{j-1}, j-1\right)
\end{array}\right]\right| .
$$

Considering the sequence of hybrid times $\left(t_{j}, j\right) \in \operatorname{dom} \tilde{\theta}$ we obtain the following upper-bound

$$
\begin{aligned}
\left|\tilde{\theta}\left(t_{j^{+}}, j^{+}\right)\right| & \leq\left|I-\gamma \frac{\sigma\left(t_{j}, j\right) \sigma\left(t_{j}, j\right)^{\top}}{\max \left\{\left|\sigma\left(t_{j}, j\right)\right|^{2}, \epsilon^{2}\right\}}\right|\left|\tilde{\theta}\left(t_{j}, j\right)\right| \\
& +\left|\frac{\gamma \sigma\left(t_{j}, j\right)}{\max \left\{\left|\sigma\left(t_{j}, t\right)\right|^{2}, \epsilon^{2}\right\}}\right|\left|\left[\begin{array}{c}
\theta \\
-e_{1}
\end{array}\right]\right|\left|\left[\begin{array}{l}
\tilde{\sigma}\left(t_{j}, j\right) \\
\tilde{\eta}\left(t_{j}, j\right)
\end{array}\right]\right| \\
& \leq\left|\tilde{\theta}\left(t_{j}, j\right)\right|+c_{4}\left|\left[\begin{array}{l}
\tilde{\sigma}\left(t_{j}, j\right) \\
\tilde{\eta}\left(t_{j}, j\right)
\end{array}\right]\right|,
\end{aligned}
$$

where the quantity $\left|\gamma \sigma / \max \left\{|\sigma|^{2}, \epsilon^{2}\right\}\right|\left|\left(\theta^{\top},-e_{1}^{\top}\right)\right|$ is globally bounded by the positive constant $c_{4}$. Applying recursively (22) into 23) we obtain the following

$$
\begin{aligned}
\left|\tilde{\theta}\left(t_{j^{+}}, j^{+}\right)\right| & \leq|\tilde{\theta}(0,0)|+c_{4} \sum_{k=0}^{j} \mathrm{e}^{-k \lambda_{2} \tau_{\min }}\left|\left[\begin{array}{l}
\tilde{\sigma}(0,0) \\
\tilde{\eta}(0,0)
\end{array}\right]\right| \\
& \leq|\tilde{\theta}(0,0)|+c_{5}\left|\left[\begin{array}{l}
\tilde{\sigma}(0,0) \\
\tilde{\eta}(0,0)
\end{array}\right]\right|
\end{aligned}
$$

which is the desired $\mathcal{K}_{\infty}$ bound. The constant $c_{5}$ is defined as $c_{5}:=c_{4} \frac{\mathrm{e}^{\lambda_{2} \tau_{\min }}}{\mathrm{e}^{\lambda_{2} \tau_{\min }-1}} \geq c_{4} \sum_{k=0}^{j} \mathrm{e}^{-k \lambda_{2} \tau_{\min }}$. From this point, the proof follows the same steps reported in the proof of Proposition 1, see (17).

\section{Simulations}

In this section we present an academic example of a second order single-input single-output plant with full relative degree ${ }^{1}$ The plant is in the form reported in (2), and is

${ }^{1}$ The example, implemented in Simulink, is available at: github.com/MatteoRagni/adaptive.right.inverse
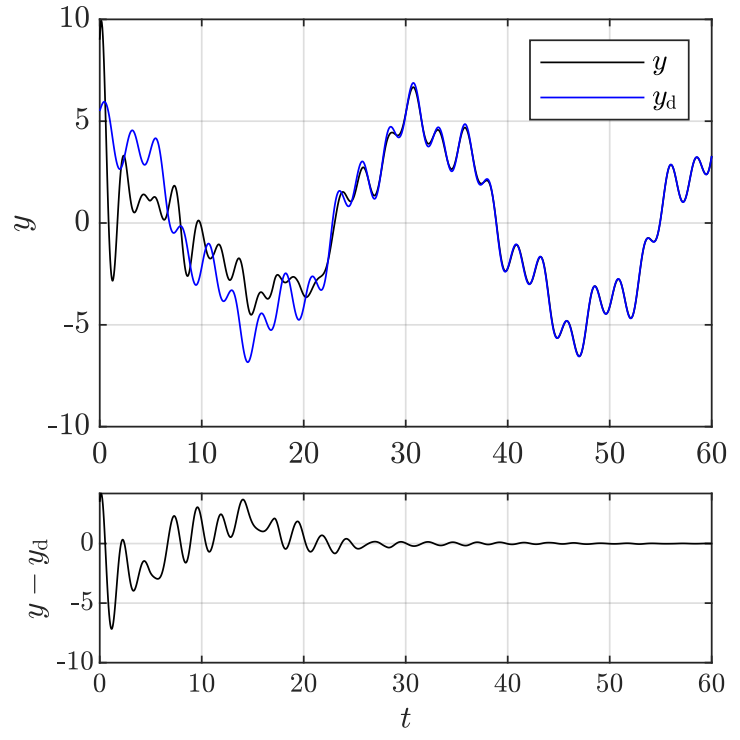

Fig. 1. Top: plant output and reference signal. Bottom: tracking error.

characterized by the following unknown matrices

$$
\left[\begin{array}{c|c}
A & B \\
\hline C &
\end{array}\right]=\left[\begin{array}{cc|c}
0 & 1 & 0 \\
-\omega_{\mathrm{n}}^{2} & -2 \zeta \omega_{\mathrm{n}} & 1 \\
\hline k & 0 &
\end{array}\right],
$$

where $k=3, \omega_{\mathrm{n}}=3$ and $\zeta=0.2$. Our objective is to asymptotically track the reference signal $y_{\mathrm{d}}(t)=$ $5 \cos (t \backslash 5)+\cos (t+\pi \backslash 3)+\sin (2 t \backslash 5)$. The pair $(\Lambda, \Phi)$ is in controllable canonical form and $\Lambda$ has been designed to ensure a large enough bandwidth to the filters. The roots of the characteristic polynomial of $\Lambda$ are reported next, $\operatorname{spec}(\Lambda)=\{-50,-75,-100\}$. The timer and adaptation parameters have been selected as follows $\tau_{\min }=0.5, \tau_{\max }=$ $3, \gamma=0.5$ and $\epsilon=1 \times 10^{-6}$. For the simulations reported in Figure 1 12 the following initial conditions have been selected $x(0,0)=(3,4)^{\top}, \sigma(0,0)=(0,0,0)^{\top}, \eta(0,0)=$ $(0,0,0)^{\top}$. The initial condition for the parameters of the inverse system have been set to $\hat{\theta}(0,0)=(1,0.2,0.1)^{\top}$, while the true value $\theta=(3,0.4,1 / 3)^{\top}$. The response of the plant output and the desired reference to track are reported in Figure 1. We can observe that after roughly 30 seconds the parameters adaptation is complete, and the tracking error $y-y_{\mathrm{d}}$ asymptotically vanishes. Figure 2 shows the $\theta, \hat{\theta}$ and $\tau$ dynamics. We can observe that the update law (19) is triggered in a non periodic fashion, but despite this fact, $\hat{\theta}$ converges to the true value $\theta$. The proof of this property will be the subject of future investigations.

\section{Conclusions}

We presented a new adaptive feed-forward control scheme that uses a novel hybrid adaptation mechanism. The scheme uses a parametric inverse of the plant to generate a feedforward control action in continuous time. Because in practice the plant, and so the inverse, are usually not well known, we rely on a hybrid adaptation mechanism that estimates the 

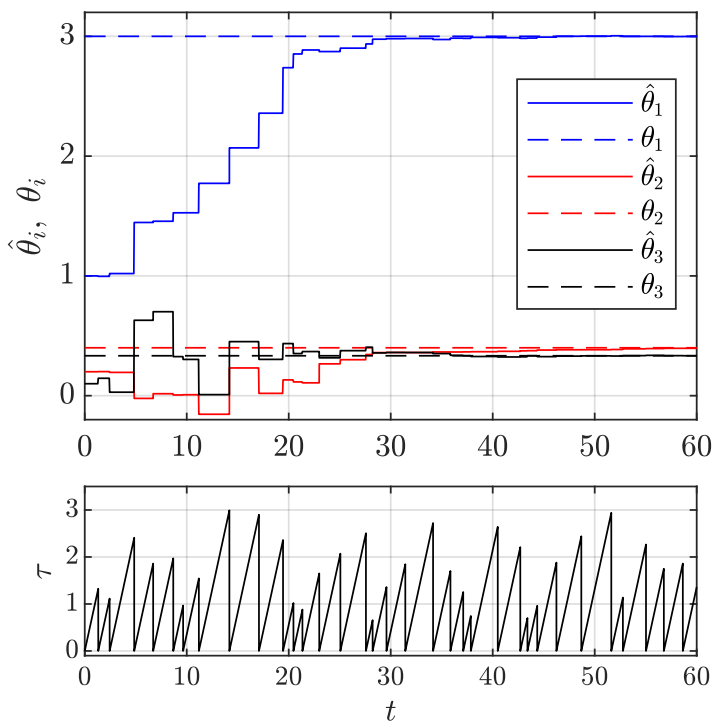

Fig. 2. Above the adaptation of $\hat{\theta}$ is plotted with solid lines, while the true value $\theta$ is plotted with dashed lines. Below we can observe one possible evolution of the timer $\tau$. Notice that the reset $\tau^{+}=0$ occurs randomly for $\tau \in\left[\tau_{\min }, \tau_{\max }\right]$.

inverse parameters. The adaptation is performed at discretetime instants so that combined with the continuous time nature of the inverse, the resulting scheme is a full hybrid system. Under mild assumptions, we proved Uniform Global Stability (UGS) for a properly chosen attractor, and we believe that this result can be strengthened to Uniform Global Asymptotic Stability (UGAS) for reference signals that satisfy a persistence of excitation property. Finally, we presented an academic example showing the potential of the proposed approach.

\section{REFERENCES}

[1] R. W. Brockett and M. Mesarović, "The reproducibility of multivariable systems," Journal of mathematical analysis and applications, vol. 11, pp. 548-563, 1965.

[2] R. W. Brockett, "The invertibility of dynamic systems with application to control," System Research Center, Case Institute of Technology, Tech. Rep., 1963.

[3] R. Brockett, "Poles, zeros, and feedback: State space interpretation," IEEE Transactions on Automatic Control, vol. 10, no. 2, pp. 129-135, 1965.

[4] G. M. Clayton, S. Tien, K. K. Leang, Q. Zou, and S. Devasia, "A review of feedforward control approaches in nanopositioning for highspeed spm," Journal of dynamic systems, measurement, and control, vol. 131, no. 6, p. 061101, 2009.

[5] C. Papageorgiou and K. Glover, "Robustness analysis of nonlinear flight controllers," Journal of Guidance, Control, and Dynamics, vol. 28, no. 4, pp. 639-648, 2005.

[6] J. Reiner, G. J. Balas, and W. L. Garrard, "Flight control design using robust dynamic inversion and time-scale separation," Automatica, vol. 32, no. 11, pp. 1493-1504, 1996.

[7] Q. Wang and R. F. Stengel, "Robust nonlinear flight control of a high-performance aircraft," IEEE Transactions on Control Systems Technology, vol. 13, no. 1, pp. 15-26, 2005.

[8] F. Lavergne, F. Villaume, G. Garcia, S. Tarbouriech, and M. Jeanneau, "Nonlinear and robust flight control laws design for the longitudinal axis of an aircraft," in Australian International Aerospace Congress, 2005. Proceedings of the 11th. Engineers Australia, 2005.
[9] P. Dorato, "On the inverse of linear dynamical systems," IEEE Transactions on systems science and cybernetics, vol. 5, no. 1, pp. 43-48, 1969.

[10] L. Silverman, "Inversion of multivariable linear systems," IEEE transactions on Automatic Control, vol. 14, no. 3, pp. 270-276, 1969.

[11] M. Sain and J. Massey, "Invertibility of linear time-invariant dynamical systems," IEEE Transactions on automatic control, vol. 14, no. 2, pp. 141-149, 1969.

[12] B. A. Francis and W. M. Wonham, "The internal model principle of control theory," Automatica, vol. 12, no. 5, pp. 457-465, 1976

[13] B. A. Francis, "The linear multivariable regulator problem," SIAM Journal on Control and Optimization, vol. 15, no. 3, pp. 486-505, 1977.

[14] A. Isidori and C. I. Byrnes, "Output regulation of nonlinear systems," IEEE transactions on Automatic Control, vol. 35, no. 2, pp. 131-140, 1990

[15] E. Gross, M. Tomizuka, and W. Messner, "Cancellation of discrete time unstable zeros by feedforward control," Journal of dynamic systems, measurement, and control, vol. 116, no. 1, pp. 33-38, 1994

[16] Q. Zou and S. Devasia, "Preview-based stable-inversion for output tracking of linear systems," Journal of dynamic systems, measurement, and control, vol. 121, no. 4, pp. 625-630, 1999.

[17] L. Marconi, G. Marro, and C. Melchiorri, "A solution technique for almost perfect tracking of non-minimum-phase, discrete-time linear systems," International Journal of Control, vol. 74, no. 5, pp. 496506, 2001.

[18] G. Marro, D. Prattichizzo, and E. Zattoni, "Convolution profiles for right inversion of multivariable non-minimum phase discrete-time systems," Automatica, vol. 38, no. 10, pp. 1695-1703, 2002.

[19] S. Devasia, D. Chen, and B. Paden, "Nonlinear inversion-based output tracking," IEEE Transactions on Automatic Control, vol. 41, no. 7, pp. 930-942, 1996.

[20] L. Hunt, G. Meyer, and R. Su, "Noncausal inverses for linear systems,' IEEE Transactions on Automatic Control, vol. 41, no. 4, pp. 608-611, 1996.

[21] L. B. Freidovich and H. K. Khalil, "Robust feedback linearization using extended high-gain observers," in Decision and Control, 2006 45th IEEE Conference on. IEEE, 2006, pp. 983-988.

[22] — , "Performance recovery of feedback-linearization-based designs," IEEE Transactions on automatic control, vol. 53, no. 10, pp. 2324-2334, 2008.

[23] D. Liberzon, A. S. Morse, and E. D. Sontag, "Output-input stability and minimum-phase nonlinear systems," IEEE Transactions on Automatic Control, vol. 47, no. 3, pp. 422-436, 2002.

[24] M. Cordioli, M. Mueller, F. Panizzolo, F. Biral, and L. Zaccarian, "An adaptive reset control scheme for valve current tracking in a powersplit transmission system." in ECC, 2015, pp. 1884-1889.

[25] F. S. Panni, H. Waschl, D. Alberer, and L. Zaccarian, "Position regulation of an egr valve using reset control with adaptive feedforward," IEEE Transactions on Control Systems Technology, vol. 22, no. 6, pp. 2424-2431, 2014.

[26] L. Zaccarian, D. Nesic, and A. R. Teel, "First order reset elements and the clegg integrator revisited," in American Control Conference, 2005. Proceedings of the 2005. IEEE, 2005, pp. 563-568.

[27] R. Goebel, R. G. Sanfelice, and A. R. Teel, Hybrid Dynamical Systems: modeling, stability, and robustness. Princeton University Press, 2012.

[28] L. Ljung and T. Söderström, Theory and practice of recursive identification. MIT press, 1983.

[29] M. Bin, L. Marconi, and A. R. Teel, "Results on adaptive output regulation for linear systems by least-squares identifiers," in 2018 IEEE Conference on Decision and Control (CDC). IEEE, 2018, pp. 1391-1396.

[30] A. Serrani, "Rejection of harmonic disturbances at the controller input via hybrid adaptive external models," Automatica, vol. 42, no. 11, pp. 1977-1985, 2006

[31] F. Forte, L. Marconi, and A. R. Teel, "Robust nonlinear regulation Continuous-time internal models and hybrid identifiers," IEEE Transactions on Automatic Control, vol. 62, no. 7, pp. 3136-3151, 2017.

[32] A. Sferlazza, S. Tarbouriech, and L. Zaccarian, "Time-varying sampled-data observer with asynchronous measurements," IEEE Transactions on Automatic Control, 2018. 